\title{
RESEARCH
}

Open Access

\section{Addressing the liver progenitor cell response and hepatic oxidative stress in experimental non-alcoholic fatty liver disease/non-alcoholic steatohepatitis using amniotic epithelial cells}

Mihiri Goonetilleke ${ }^{1,2}$, Nathan Kuk ${ }^{1,3}$, Jeanne Correia ${ }^{1,2,3}$, Alex Hodge ${ }^{1,3}$, Gregory Moore ${ }^{1,3}$, Michael P. Gantier ${ }^{4,5}$, George Yeoh ${ }^{6,7,8}$, William Sievert ${ }^{1,3+}$ and Rebecca Lim ${ }^{2,9^{*}+}$ (D)

\begin{abstract}
Background: Non-alcoholic fatty liver disease is the most common liver disease globally and in its inflammatory form, non-alcoholic steatohepatitis (NASH), can progress to cirrhosis and hepatocellular carcinoma (HCC). Currently, patient education and lifestyle changes are the major tools to prevent the continued progression of NASH. Emerging therapies in NASH target known pathological processes involved in the progression of the disease including inflammation, fibrosis, oxidative stress and hepatocyte apoptosis. Human amniotic epithelial cells (hAECs) were previously shown to be beneficial in experimental models of chronic liver injury, reducing hepatic inflammation and fibrosis. Previous studies have shown that liver progenitor cells (LPCS) response plays a significant role in the development of fibrosis and HCC in mouse models of fatty liver disease. In this study, we examined the effect hAECs have on the LPC response and hepatic oxidative stress in an experimental model of NASH.

Methods: Experimental NASH was induced in C57BL/6 J male mice using a high-fat, high fructose diet for 42 weeks. Mice received either a single intraperitoneal injection of $2 \times 10^{6}$ hAECs at week 34 or an additional hAEC dose at week 38. Changes to the LPC response and oxidative stress regulators were measured.

Results: hAEC administration significantly reduced the expansion of LPCs and their mitogens, IL-6, IFNY and TWEAK. hAEC administration also reduced neutrophil infiltration and myeloperoxidase production with a concurrent increase in heme oxygenase-1 production. These observations were accompanied by a significant increase in total levels of anti-fibrotic IFN $\beta$ in mice treated with a single dose of hAECs, which appeared to be independent of cGAS-STING activation.
\end{abstract}

\footnotetext{
* Correspondence: Rebecca.Lim@monash.edu

+William Sievert and Rebecca Lim contributed equally to this work.

${ }^{2}$ The Ritchie Centre, Hudson Institute of Medical Research, Melbourne, Victoria, Australia

${ }^{9}$ Department of Obstetrics and Gynaecology, Monash University, Melbourne, Victoria, Australia

Full list of author information is available at the end of the article
}

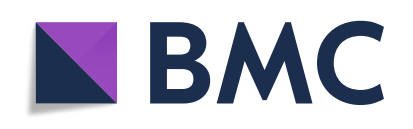

(- The Author(s). 2021 Open Access This article is licensed under a Creative Commons Attribution 4.0 International License, which permits use, sharing, adaptation, distribution and reproduction in any medium or format, as long as you give appropriate credit to the original author(s) and the source, provide a link to the Creative Commons licence, and indicate if changes were made. The images or other third party material in this article are included in the article's Creative Commons licence, unless indicated otherwise in a credit line to the material. If material is not included in the article's Creative Commons licence and your intended use is not permitted by statutory regulation or exceeds the permitted use, you will need to obtain permission directly from the copyright holder. To view a copy of this licence, visit http://creativecommons.org/licenses/by/4.0/. The Creative Commons Public Domain Dedication waiver (http://creativecommons.org/publicdomain/zero/1.0/) applies to the data made available in this article, unless otherwise stated in a credit line to the data. 
Conclusions: Expansion of liver progenitor cells, hepatic inflammation and oxidative stress associated with experimental NASH were attenuated by hAEC administration. Given that repeated doses did not significantly increase efficacy, future studies assessing the impact of dose escalation and/or timing of dose may provide insights into clinical translation.

Keywords: Fatty liver disease, NASH/NAFLD, Liver progenitor cells, Hepatic oxidative stress, Amnion epithelial cells, Regenerative medicine

\section{Background}

Non-alcoholic fatty liver disease (NAFLD) is the most common liver disease globally [1]. While global prevalence of NAFLD varies widely, the incidence rates in some countries are as high as $45 \%$ [2]. As many as one third of those affected by NAFLD may progress to inflammation-associated fibrotic disease and cirrhosis (non-alcoholic steatohepatitis; NASH) [3] and eventually hepatocellular carcinoma (HCC). The global prevalence of NAFLD is currently estimated at 25\% [4], and in 2020, NASH replaced hepatitis $\mathrm{C}$ as the leading reason for liver transplantation. While patient education and lifestyle changes are considered the major tools to prevent progression of liver disease, there is currently no cure for NAFLD/NASH. With the failure of several drugs in late stage clinical development and for patients who are not transplantable, alternative options must be explored.

Emerging therapies in NASH target known pathological processes and pathways involved in the progression of the disease including inflammation, fibrosis, oxidative stress and apoptosis. Given previous reports suggesting that liver progenitor cells (LPCs) are cellular targets for malignant transformation in hepatocellular carcinoma (HCC), and the increased risk of HCC in NALFD/NASH [5-9], LPCs have been identified as a possible target for treatment [10]). Furthermore, antioxidants, including N-Acetylcysteine (NAC) and Sadenosylmethionine (SAMe), have been explored as potential treatment for NASH [11-13] since oxidative stress has been implicated in the progression of the LPC response [14] and pathogenesis of NASH. Recent preclinical studies have also explored interferon $\beta$ (IFN $\beta$ ) as a potential anti-fibrotic for $\mathrm{NASH}$, with its ability to downregulate fibrogenic genes associated with $T G F \beta-1$ and $M y D 88$ pathways [15]. While these emerging treatments have shown varying degrees of success, there remains an urgent need to develop efficacious therapies that address the complex pathophysiologic processes implicated in NASH.

Cell-based therapies have shown promising results in the treatment and prevention of experimental NAFLD/ NASH $[16,17]$. Human amnion epithelial cells (hAEC) that line the amniotic sac of the placenta are nontumorigenic and immunologically privileged. The therapeutic potential of hAECs has been explored in the setting of liver [18], lung [19], cardiac [20], epidermal [21] and neurological injury [22]. We [16, 18, 23-26] and others $[27,28]$ postulate that hAECs may be a promising alternative to address NAFLD/NASH either through the restriction of hepatocyte death and hepatic stellate cell activation [24] and/or through the modification of dominant macrophage phenotyp e[18, 24]. Both hAECs and their secretome have been shown to reduce chronic liver injury [23, 24]. Kuk et al. previously reported a reduction in hepatic inflammation and fibrosis in this model of experimental NASH when treated with hAECs [24]. While the anti-inflammatory and antifibrotic effects of hAECs have been extensively explored in carbon tetrachloride models of liver injury [18, 2325], their effect on liver regeneration in the context of NAFLD/NASH, is poorly understood.

In this study, we investigate the influence hAECs have on the LPC response and hepatic oxidative stress in a murine model of human NASH. Previous reports show that hAECs dampen hepatic inflammation and fibrosis in experimental NASH [24], but the mechanisms through which hAECs and other cell-based modalities influence the LPC response and oxidative stress response remain unknown.

\section{Methods \\ Ethics statement}

This study was approved by Monash University Animal Ethics Committee (AE\# B13/01) and conducted in accordance with the Australian Code of Practice for the Care and Use of Animals for Scientific Purposes (2006). All mice were monitored daily. The Monash Health Human Research Ethics Committee approved the collection and use of human amnion (Monash Health HREC approval numbers: 01067B, 12223B). Informed written consent was obtained from each patient prior to surgery.

\section{Isolation of hAECs}

The hAECs were isolated as previously described [29]. Briefly, amniotic membranes were separated from underlying chorions, washed in Hanks Balanced Salt Solution (HBSS) and digested in 0.05\% trypsin-EDTA (Thermo Fisher Scientific, Waltham, MA) for $1 \mathrm{~h}$ at $37^{\circ} \mathrm{C}$. Only batches with $>90 \%$ cellular viability were 
cryopreserved in liquid nitrogen for use in this study. The purity of hAEC isolates were determined by flow cytometry with EpCAM (Becton Dickinson Biosciences, Bedford, MA), CD90 (BioLegend, San Diego, CA), CD45 (Invitrogen, Carlsbad, CA) and CD31 (Becton Dickinson Biosciences, Bedford, MA) used to assess cell purity. Only batches with $>90 \%$ EpCAM (Supplemental Figure 1a) and $<1 \%$ CD90, CD45 and CD31 (Supplemental Figure $1 \mathrm{~b}, \mathrm{c}$ and $\mathrm{d}$ ) positive cells were used for the following experiments. hAECs isolated from 4 placental donors were used for this animal study.

\section{Animals and experimental schedule}

Seven-week-old male C57BL/6 J mice were purchased from Monash Animal Services (Monash University, Melbourne, Australia). Mice were divided into four groups ( $n=6-8)$, one group receiving ad libitum standard chow with normal water (normal), and the other three groups receiving a modified experimental NASH diet with high fructose corn syrup for 42 weeks (Table 1 ). The three groups receiving the experimental NASH diet were either on the diet alone (FF), received a single intraperitoneal injection of $2 \times 10^{6}$ hAECs (FFHS) at week 34 or received two doses of $2 \times 10^{6}$ hAECs (FFHD) at week 34 and 38 (Fig. 1). Control mice included standard chow fed and mice on the experimental NASH diet alone (FF). All mice were culled at week 42 .

\section{Immunohistochemistry and immunofluorescence}

Paraffin-embedded liver sections from all treatment groups were dewaxed, rehydrated and incubated in 10 $\mathrm{mM}$ sodium citrate $\mathrm{pH} 6$ or $10 \mathrm{mM}$ Tris-EDTA pH 9 for heat mediated antigen retrieval (Table 2). Endogenous peroxidase activity was blocked using $3 \% \mathrm{H}_{2} \mathrm{O}_{2}$. Tissue sections were blocked with a universal protein blocking solution for $1 \mathrm{~h}$ and then incubated with primary antibodies overnight at $4{ }^{\circ} \mathrm{C}$ as per Table 2 . Tissue sections were then washed three times and incubated with secondary antibodies (Table 2) for $1 \mathrm{~h}$. For PanCK, antibody binding was detected using Vectastain $A B C$ HRP kit
(Vector Laboratories, Meadowbrook, QLD, Australia) followed by DAB chromogen (Dako, Mulgrave, Victoria, Australia). Sections for MPO, CD45, NIMP-R14 and HO-1 were incubated with DAPI (Sigma-Aldrich, St. Louis, MO, USA) for $10 \mathrm{~min}$. Data are presented as number of cells per field of view (PanCK) or percentage (\%) positive cells per five non-overlapping fields at $\times 20$ magnification (CD45, NIMP-R14, MPO and HO-1) normalised to the number of DAPI positive cells using Image J (v1.53c, National Institutes of Health, USA).

\section{RNA isolation and RT-PCR}

Total RNA was isolated from mouse liver tissue or cultured cells using the RNeasy mini-kit according to the manufacturer's instructions (Qiagen Pty Ltd, Hilden, Germany). cDNA was synthesised using the HighCapacity Reverse Transcription Kit (Applied Biosystems, CA, USA) and amplified using Power SYBR ${ }^{\mathrm{m} w}$ Green PCR Master Mix (Applied Biosystems, CA, USA) for qPCR. Quantitative RT-PCR (QuantStudio Real-time PCR system) was used to examine gene expression levels of Nox2, Nox4, Sting, Il-6, Tweak, Ifn $\gamma$, Ifn $\beta$, Rsad2, Ifit1, Ifih1 and Isg15 (Table 3). Data were normalised to the housekeeping gene $18 \mathrm{~s}$ with fold change calculated using delta cycle-threshold method [30]. Melting curves were used in each run to confirm specificity of amplification.

\section{iMACs and BMOLs co-cultured with hAECS}

Immortalised mouse macrophages (iMACs) and bipotential murine oval liver cells (BMOLs), a mouse LPC cell line, were co-cultured with hAECs from 4 donors, in $0.4 \mu \mathrm{m}$ transwell inserts, in 6-well plates at a 1:5 ratio. Wells containing only iMACs or BMOLs served as negative controls and were maintained in DMEM:F12 supplemented with 5-10\% FBS or DMEM:F12 supplemented with $30 \mathrm{ng} / \mathrm{mL}$ IGF-II, $50 \mathrm{ng} / \mathrm{mL}$ EGF, $10 \mu \mathrm{g} / \mathrm{mL}$ insulin, $100 \mathrm{U} / \mathrm{mL}$ penicillin and streptomycin and $5-10 \%$ FBS, respectively [26]. Cells treated with 5,6-dimethylxanthenone-4-acetic acid (DMXAA-D5817, Sigma-Aldrich, St.

Table 1 Nutritional composition of the experimental NASH diet and standard chow

\begin{tabular}{lll}
\hline Dietary composition & Standard chow & Fast food diet \\
\hline Total fat (\% weight) & $4.8 \%$ & $21 \%$ \\
Saturated & $0.93 \%$ & $14 \%$ \\
Mono-unsaturated & $0.99 \%$ & $6.23 \%$ \\
Polyunsaturated & $2.20 \%$ & $0.77 \%$ \\
Cholesterol (\% weight) & $0 \%$ & $2 \%$ \\
Carbohydrates (\% weight) & $59.4 \%$ & $49.9 \%$ \\
Protein (\% weight) & $20 \%$ & $21 \%$ \\
Fibre (\% weight) & $4.7 \%$ & $4.7 \%$ \\
Drinking water & Tap water & High fructose water $42 \mathrm{~g} / \mathrm{L}) 55 \%$ fructose, 45\% sucrose \\
\hline
\end{tabular}




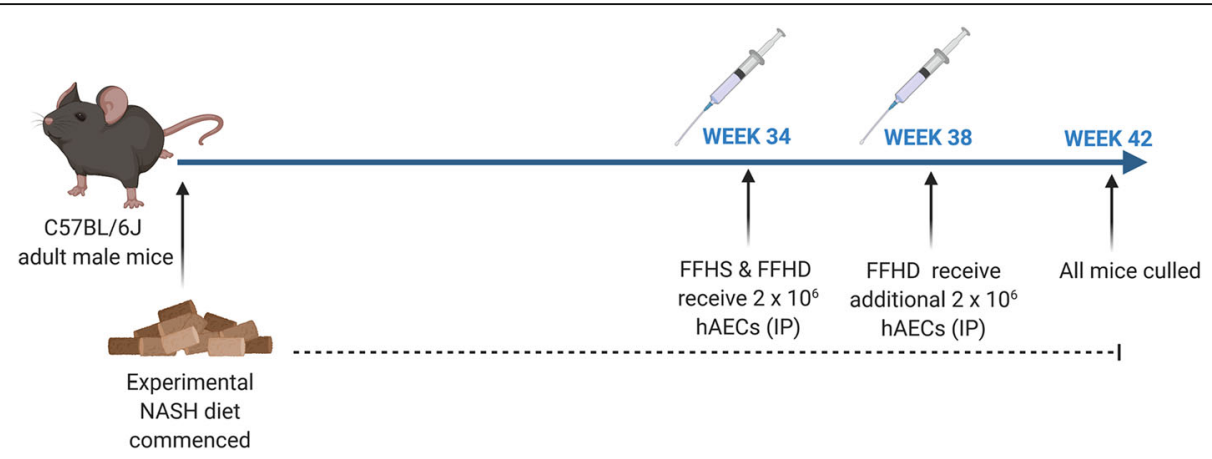

Fig. 1 Schedule for experimental NASH diet and hAEC administration. Experimental NASH was induced in C57BL/6 J male mice using a high-fat, high fructose diet for 42 weeks. Mice received either a single intraperitoneal injection of $2 \times 10^{6}$ hAECs at week 34 or an additional hAEC dose at week 38

Louis, MO, USA) served as positive controls for cGASSTING activation. Cells treated with inflammatory cytokines IFNY and TNF $\alpha, 24 \mathrm{~h}$ before hAEC treatment, served as controls for inflammation. Cultures were maintained at $37^{\circ} \mathrm{C}$ in $95 \%$ humidity and $5 \% \mathrm{CO}_{2}$ for $2 \mathrm{~h}$ prior to RNA isolation.

\section{Data analysis}

Data were analysed using GraphPad Prism version 6.0 software (GraphPad Software, San Diego, CA, USA). Murine studies were conducted with 6-8 animals in each group. One way analysis of variance with Dunn's post-hoc test for multiple comparisons was performed. Differences were considered statistically significant when $p<0.05$. Data are presented as mean \pm standard error of mean.

\section{Results}

hAECs reduced LPC response in experimental nonalcoholic steatohepatitis

The experimental NASH diet significantly increased (5.5-fold) the number of LPCs (PanCK +cells) in the liver (Normal vs. FF; $21.47 \pm 0.18$ vs. $117.8 \pm 11.5$, Fig. $2 \mathrm{a}, p<0.0001)$. A single dose of hAECs significantly decreased the number of LPCs (FF vs. FFHS; $117.8 \pm 11.5$ vs. $76.27 \pm 5.9$, Fig. $2 \mathrm{a}, p=0.005)$, but no greater reduction was seen in the group that received a second dose of hAECs. It is worth noting that hAEC administration did not return LPC numbers to control levels. The number of PanCK+ cells in the FFHS and FFHD groups remained elevated compared to healthy controls (normal vs. FFHS; 21.47 \pm 0.18 vs. $76.27 \pm 5.9$, Fig. $2 \mathrm{a}, p=0.0088$; normal vs. FFHD; $21.47 \pm 0.18$ vs. $91.07 \pm 8.07$, Fig. $2 \mathrm{a}, p=$ 0.0019).

Next, we assessed changes to transcriptional levels of known LPC mitogens in whole mouse liver tissue. Here, we observed that compared with FF mice, a single dose of hAEC reduced transcription of $\mathrm{Il}-6$ by 20 -fold (FFHS vs. FF; $0.06 \pm 0.03$ vs. $1.2 \pm 0.27$, Fig. $2 \mathrm{~b}, p=0.001$ ) and by 24 -fold in the FFHD group (FFHD vs. FF; $0.05 \pm 0.029$ vs. $1.2 \pm 0.27$, Fig. $2 b, p$ $=0.002)$. The transcription of Tweak was reduced by 7 -fold in the FFHS group $(0.17 \pm 0.06$ vs. $1.2 \pm 0.24$, Fig. 2c, $p=0.001$ ) and was 5-fold lower in the FFHD group $(0.22 \pm 0.9$ vs. $1.2 \pm 0.24$, Fig. $2 \mathrm{c}, p=0.002)$ compared to the fast food only group. Furthermore, transcriptional levels of Ifny were 23-fold lower in the FFHS group $(0.04 \pm 0.009$ vs. $0.94 \pm 0.2$, Fig. $2 \mathrm{~d}, p=$ $0.0003)$ and 15 -fold lower in the FFHD group (0.06 \pm 0.02 vs. $0.94 \pm 0.2$, Fig. $2 d, p=0.0007$ ) compared to the FF group.

Table 2 Immunohistochemistry and immunofluorescence antigen retrieval, primary and secondary antibodies

\begin{tabular}{lll}
\hline Antigen retrieval & Primary antibody & Secondary antibody \\
\hline $\begin{array}{l}\text { 10 mM sodium } \\
\text { citrate (pH 6) }\end{array}$ & $\begin{array}{l}\text { Wide spectrum screening cytokeratin (Pan-CK) (ZO622, Dako, } \\
\text { Mulgrave, Victoria, Australia, 1:200) }\end{array}$ & $\begin{array}{l}\text { Biotinylated goat anti-rabbit IgG (BA-100, Vector Laboratories, } \\
\text { Meadowbrook, QLD, Australia, 1:150) }\end{array}$ \\
& $\begin{array}{l}\text { Rabbit myeloperoxidase (MPO) (ab45977, Abcam, Cambridge, } \\
\text { MA, USA, 1:100) }\end{array}$ & $\begin{array}{l}\text { Donkey anti-rabbit 568 (Alexa Fluor conjugates, Life Technolo- } \\
\text { gies, Frederick, MD, USA, 1:500) }\end{array}$ \\
& Rabbit CD45 (ab10558, Abcam, Cambridge, MA, USA, 1:100) & $\begin{array}{l}\text { Goat anti-rabbit 647 (Alexa Fluor conjugates, Life Technologies, } \\
\text { Frederick, MD, USA, 1:500) }\end{array}$ \\
$\begin{array}{l}\text { Tris-EDTA buffer } \\
\text { (pH 9) }\end{array}$ & $\begin{array}{l}\text { Rat anti-neutrophil (NIMP-R14) } \\
\text { (ab2557, Abcam, Cambridge, MA, USA, 1:100) }\end{array}$ & $\begin{array}{l}\text { Goat anti-rat 488 (Alexa Fluor conjugates, Life Technologies, } \\
\text { Frederick, MD, USA, 1:100) }\end{array}$ \\
& $\begin{array}{l}\text { Rabbit recombinant anti-heme oxygenase 1 (HO-1) } \\
\text { (ab52947, Abcam, Cambridge, MA, USA, 1:100) }\end{array}$ & $\begin{array}{l}\text { Donkey anti-rabbit 568 (Alexa Fluor conjugates, Life Technolo- } \\
\text { gies, Frederick, MD, USA, 1:500) }\end{array}$ \\
\hline
\end{tabular}


Table 3 Real-time quantitative PCR primers

\begin{tabular}{|c|c|c|}
\hline & PCR primers & Sequence \\
\hline \multirow[t]{24}{*}{ MOUSE } & $m$ Nox2-FWD & TGT CAT TCT GGT GTG GTT GG \\
\hline & mNox2-REV & GCA GCA GGA TCA GCA TAC AG \\
\hline & mNox4-FWD & CCA GAA TGA GGA TCC CAG AA \\
\hline & mNox4-REV & ACC ACC TGA AAC ATG CAA CA \\
\hline & mSting-FWD & CTA CAT TGG GTA CTT GCG GTT \\
\hline & mSting-REV & GCA CCA CTG AGC ATG TTG TTA TG \\
\hline & $m \|-6-F W D$ & ATG GAT GCT ACC AAA CTG GAT \\
\hline & mII-6-REV & TGA AGG ACT CTG GCT TTG TCT \\
\hline & mTweak-FWD & TTG GCC TCC TGC TGG TCG TGG TCA \\
\hline & mTweak-REV & CTC CCG GCG GTC CTC TGC TGT CA \\
\hline & mlfny-FWD & GCG TCA TTG AAT CAC ACC TG \\
\hline & $m I f n \gamma-R E V$ & TGA GCT CAT TGA ATG CTT GG \\
\hline & $m R n 18 s-F W D$ & GTA ACC CGT TGA ACC CCA TT \\
\hline & mRn18s-REV & CCA TCC AAT CGG TAG TAG CG \\
\hline & mlfit1-RT-FWD & GAG AGT CAA GGC AGG TाT CT \\
\hline & mlfit1-RT-REV & TCT CAC TTC CAA ATC AGG TAT GT \\
\hline & mlfn $\beta 1$-FWD & CCC TAT GGA GAT GAC GGA GA \\
\hline & mlfn $\beta 1-R E V$ & CCC AGT GCT GGA GAA ATT GT \\
\hline & mRsad2-FWD & CTG TGC GCT GGA AGG TाT \\
\hline & mRsad2-REV & ATT CAG GCA CCA AAC AGG AC \\
\hline & $\mathrm{m} / \mathrm{sg} 15-\mathrm{FWD}$ & CAA TGG CCT GGG ACC TAA AG \\
\hline & m/sg15-REV & TAA GAC CGT CCT GGA GCA CT \\
\hline & mlfih1-FWD & TCT TGG ACA CTT GCT TCG AG \\
\hline & mlfih1-REV & TCC TTC TGC ACA ATC CTT CTC \\
\hline
\end{tabular}

\section{hAECs reduced neutrophil infiltration and hepatic oxidative stress}

Given previous reports on the role of neutrophil infiltration [31] and the central role of oxidative stress in $\mathrm{NASH}$, we next evaluated the effect of hAECs on these aspects of liver pathology. We observed a significant increase in leukocytes (CD45+ cells) in the experimental model of NASH (FF vs. normal; $37.9 \pm 2.9 \%$ vs. $21.3 \pm$ $1.08 \%$, Fig. 3a, $p<0.0001)$. Furthermore, significantly lower levels of CD45+ cells were observed in the FFHS group $(23.25 \pm 1.03 \%$ vs. $21.3 \pm 1.08 \%$, Fig. $3 \mathrm{a}, p<$ $0.0001)$ and the FFHD group $(29.61 \% \pm 1.7$ vs. $21.3 \pm$ $1.08 \%$, Fig. 3a, $p=0.0076)$ compared to the FF group. Notably, numbers of CD45+ cells were significantly lower in the FFHS compared to the FFHD group (23.25 $\pm 1.03 \%$ vs. $29.61 \% \pm 1.7$, Fig. $3 \mathrm{a}, p=0.001)$. Further, we noted a significant increase in NIMP-R14+ neutrophils in the FF group compared to the control group (17.8 \pm $1.7 \%$ vs. $1.2 \pm 0.2 \%$, Fig. $3 \mathrm{~b}, p<0.0001)$. Compared to the FF group, the number of NIMP-R14+ neutrophils was significantly reduced in both the FFHS (10.81 \pm $0.8 \%$ vs. $17.8 \pm 1.7 \%, p=0.0001)$ and FFHD group $(9.3 \pm$ $0.9 \%$ vs. $17.8 \pm 1.7 \%$, Fig. $3 \mathrm{~b}, p<0.0001)$. This coincided with changes in hepatic MPO levels, where we observed a significant increase in the number of $\mathrm{MPO}+$ cells compared to control $(62.7 \pm 7.7 \%$ vs. $10.3 \pm 1.8 \%$, Fig. 3 c, $p<$ $0.0001)$. Further, the number of $\mathrm{MPO}+$ cells was significantly reduced in the FFHS (29.3 $\pm 3.5 \%$ vs. $62.7 \pm 7.7 \%$, Fig. 3c, $p<0.0001)$ and FFHD groups $(23.7 \pm 3.08 \%$ vs. $62.7 \pm 7.7 \%$, Fig. 3c, $p<0.0001)$.

We further investigated the effect of hAECs on oxidative stress by assessing the relative expression of Nox4 and Nox2. We observed a 6-fold reduction in the expression levels of NOX2 in the FFHS group ( $0.2 \pm 0.1$ vs. 1.2 \pm 0.22 , Fig. $4 \mathrm{a} p=0.003)$ and a 4.8 -fold reduction in the FFHD group $(0.25 \pm 0.13$ vs. $1.2 \pm 0.22$, Fig. $4 \mathrm{a}, p=$ 0.005). While transcriptional levels of NOX4 were lower in hAEC treated mice, this difference was not statistically significant (Fig. 4b, $p=0.10$ ). We next assessed HO-1 production and found that, compared to the FF only group, there was a significant increase in expression of HO- 1 in mice treated with a single dose of hAECs $(20.72 \pm 2.1$ vs. $30.8 \pm 1.9 \%$, Fig. 4 c, $p<0.0001)$ but not a double dose of hAECs. Notably, there was no significant difference in $\mathrm{HO}-1+$ cells between the FFHD and FF group.

\section{hAECs increased total levels of hepatic IFN $\beta$}

Hepatic levels of IFN $\beta$ were increased by 4 -fold in the FFHS group compared to the FF group $(1.3 \pm 0.34$ vs. $5.6 \pm 1.8$, Fig. $5 \mathrm{a}, p=0.005)$. Interestingly, this was not observed in the FFHD mice, with a significant difference in the hepatic levels of IFN $\beta$ between the FFHS and FFHD groups $(5.5 \pm 1.8$ vs. $1.9 \pm 1.3$, Fig. $5 \mathrm{a}, p=0.03)$. No significant differences were observed in the expression of several IFN-induced genes (Rsad2, Ifit1, Isg15 and Ifih1) between treatment groups (data not shown). Furthermore, the expression of STING in the FFHD group was 10-fold higher than the FF group (36.6 \pm 8.7 vs. $3.5 \pm 0.8$, Fig. $5 \mathrm{~b}, p<0.0001)$. In order to investigate whether the increase in hepatic IFN- $\beta$ in the FFHS group was due to a direct effect on c-GAS-STING, we performed a series of in vitro studies, co-culturing hAECs with iMACs or BMOLs. Here, we observed that co-culturing with hAECs did not significantly alter the expression of IFIT1 (Fig. 5c), IFN $\beta$ (Fig. 5d) and RSAD2 (Fig. 5e) in iMACs. Similarly, co-culturing with hAECs did not significantly alter the expression of IFIT1 (Fig. 5f), IFN $\beta$ (Fig. 5g) and RSAD2 (Fig. 5h) in BMOLs. These findings suggest that the increase in hepatic IFN$\beta$ expression in the FFHS group was unlikely to be due to a direct effect of hAECs on iMACs or BMOLs-the major responders to cGAS-STING signalling in the liver.

\section{Discussion}

The main objective of this study was to determine the impact of hAECs on the LPC population and hepatic 

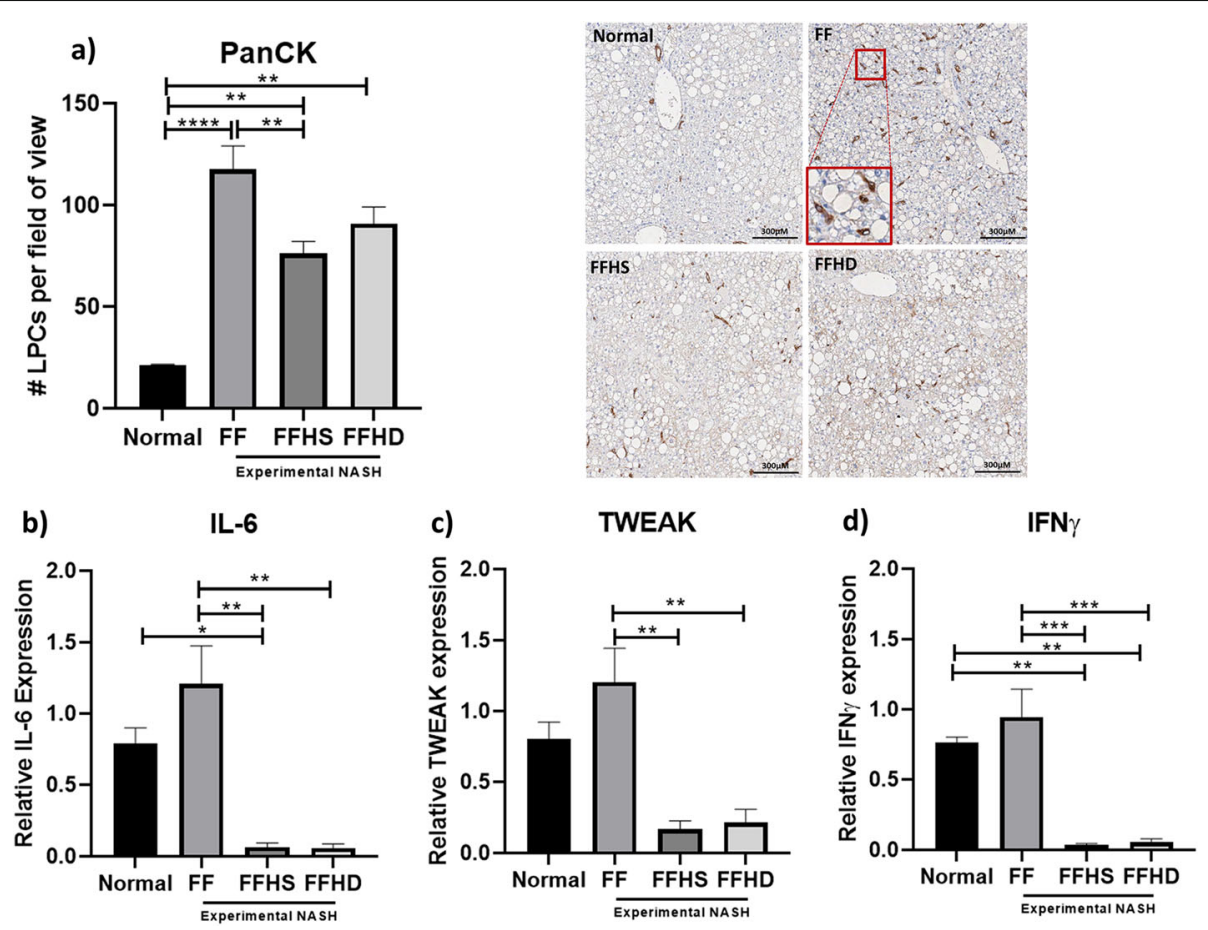

Fig. 2 The effect of hAECs on liver progenitor cell numbers and liver progenitor cell mitogens, IL-6, TWEAK and IFNy. All mice on the NASH diet, (FF, FFHS and FFHD) had a significantly greater number of liver progenitor cells (LPC) compared with normal mice (a). Within the NASH diet groups, mice treated with a single dose of hAECS (FFHS) had significantly fewer LPCs than FF mice (a). Compared with FF mice, FFHS and FFHD mice had significantly lower expression levels of II-6 (b), Tweak (c), and Ifny (d). Compared with normal mice, II-6 gene expression was significantly lower in FFHS mice (b) and Ifny gene expression was significantly lower in mice treated with both a single and double dose of hAECs (d). Magnification: $\times 10$, scale bar $=300 \mu \mathrm{m}$; red box indicating selected area at $\times 20$ magnification $(\mathbf{a}) .{ }^{*} P<0.05,{ }^{* *} P<0.01,{ }^{* * *} P<0.001$. FF, NASH diet alone; FFHD, NASH diet with double hAEC dose; FFHS, NASH diet with single hAEC dose

oxidative stress, in a murine model of human NASH. Previous studies report that that hAECs dampen hepatic inflammation and fibrosis in experimental NASH [24], but the mechanisms through which hAECs, and other cell-based modalities influence the LPC response remain unknown. Previously, Kuk et al. reported that hAEC administration reduced hepatic fibrosis by decreasing activation of hepatic stellate cells and macrophages involved in inflammation and fibrogenesis in a murine model of human NASH. hAEC treatment reduced hepatic fibrosis area, which was associated with a reduction in the number of activated hepatic stellate cells and reduced hepatic macrophage numbers. While hAECs significantly reduced hepatic inflammation and fibrosis, they did not affect the metabolic components of NASH. Kuk et al. reported no significant change in the NASH Activity Score, total cholesterol levels, serum ALT or glucose tolerance tests. Kuk et al. suggested that the hAECs had no therapeutic effect on blood glucose levels due to reduced insulin sensitivity, inherent in the experimental NASH model as it is characterised by insulin resistance. They further highlighted that previous studies on NAFLD/ NASH have not shown a reduction in serum cholesterol levels or bodyweight, suggesting that as the diet remained constant throughout the experimental period, a significant change in bodyweight was not expected [24]. In this study, we demonstrate that hAECs dampen the LPC response in experimental NASH through the reduction of critical LPC mitogens including TWEAK, IL-6 and IFN $\gamma$. Interestingly, we also observed that hAEC administration reduced leucocyte and specifically neutrophil infiltration and myeloperoxidase production with concurrent increase in $\mathrm{HO}-1$ production. This observation was accompanied by an increase in total levels of anti-fibrotic IFN $\beta$ which appeared to be independent of c-GAS-STING activation.

Liver progenitor cells are facultative stem cells that reside in the Canals of Hering. This niche is activated during chronic liver injury when hepatocyte proliferation is insufficient to achieve homeostasis. In a fibrogenic environment where TGF $\beta$ levels are persistently elevated, the balance is tipped towards LPC expansion as hepatocytes undergo TGF $\beta$-induced apoptosis $[32,33]$. The expansion of LPCs is mediated by mitogens that significantly overlap with pro-inflammatory cytokines released by hepatic macrophages, including tumour necrosis factor (TNF), lymphotoxin $\beta$ (LT $\beta)$, interferon $\gamma$ $(\mathrm{IFN} \gamma$ ), interleukin 6 (IL-6) and tumour necrosis factor- 

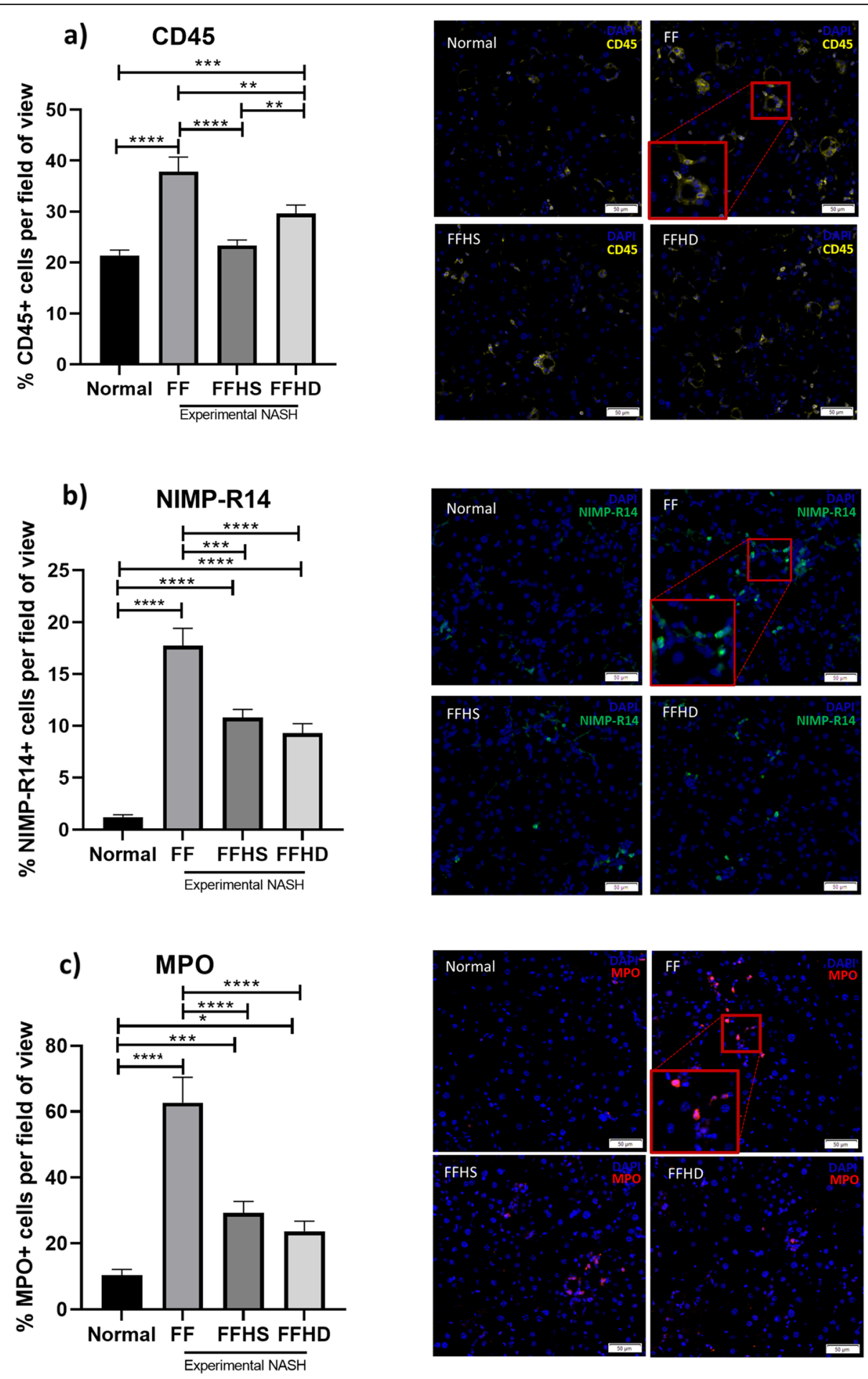

Fig. 3 (See legend on next page.) 
(See figure on previous page.)

Fig. 3 hAECs reduce levels of hepatic leukocytes, neutrophil infiltration and myeloperoxidase production. Compared with normal mice, mice on the NASH diet only (FF) and mice treated with a double dose of hAECS (FFHD) had significantly more hepatic leukocytes (CD45+ cells) (a). Within the NASH diet groups, both FFHS and FFHD mice had significantly lower levels of hepatic leukocytes compared to untreated FF mice (a). Within the mice treated with hAECS, FFHS mice had significantly fewer hepatic leukocytes compared to FFHD mice (a). Compared with normal mice, mice on the NASH diet, had significantly more neutrophils (NIMP-R14+ cells) (b). Within the NASH diet groups, FFHS and FFHD mice had significantly lower levels of neutrophils compared to untreated FF mice (b). Compared with normal mice, mice on the NASH diet, (FF, FFHS and FFHD) had significantly greater levels of MPO activity (MPO+ cells) (c). Within the NASH diet groups, FFHS and FFHD mice had significantly lower levels of MPO activity compared to untreated FF mice $(\mathbf{c})$. Magnification: $\times 10$, scale bar $=50 \mu \mathrm{m}$; red box indicating selected area at $\times 20$ magnification. ${ }^{* *} P<0.01,{ }^{* *} P<0.001,{ }^{* * * P}<0.0001 . \mathrm{MPO}$, myeloperoxidase

like weak inducer of apoptosis (TWEAK) $[5,7,26,34-$ 37]. We have shown a reduction in both LPC numbers and expression levels of the LPC mitogens IL-6, IFNY and TWEAK in hAEC treated mice most likely related to a concomitant reduction in activated hepatic macrophages. While hAECs increased LPC proliferation in in vitro co-culture studies [26], the reduction in LPC response was again most likely achieved by hAEC reduction of hepatic macrophages [24] and their secreted LPC mitogens. We have observed this consistently in previous studies using in vivo liver and lung injury models that have demonstrated that hAECs reduce macrophage recruitment and support a predominant alternatively activated (M2) phenotype $[18,19]$. It should be noted that
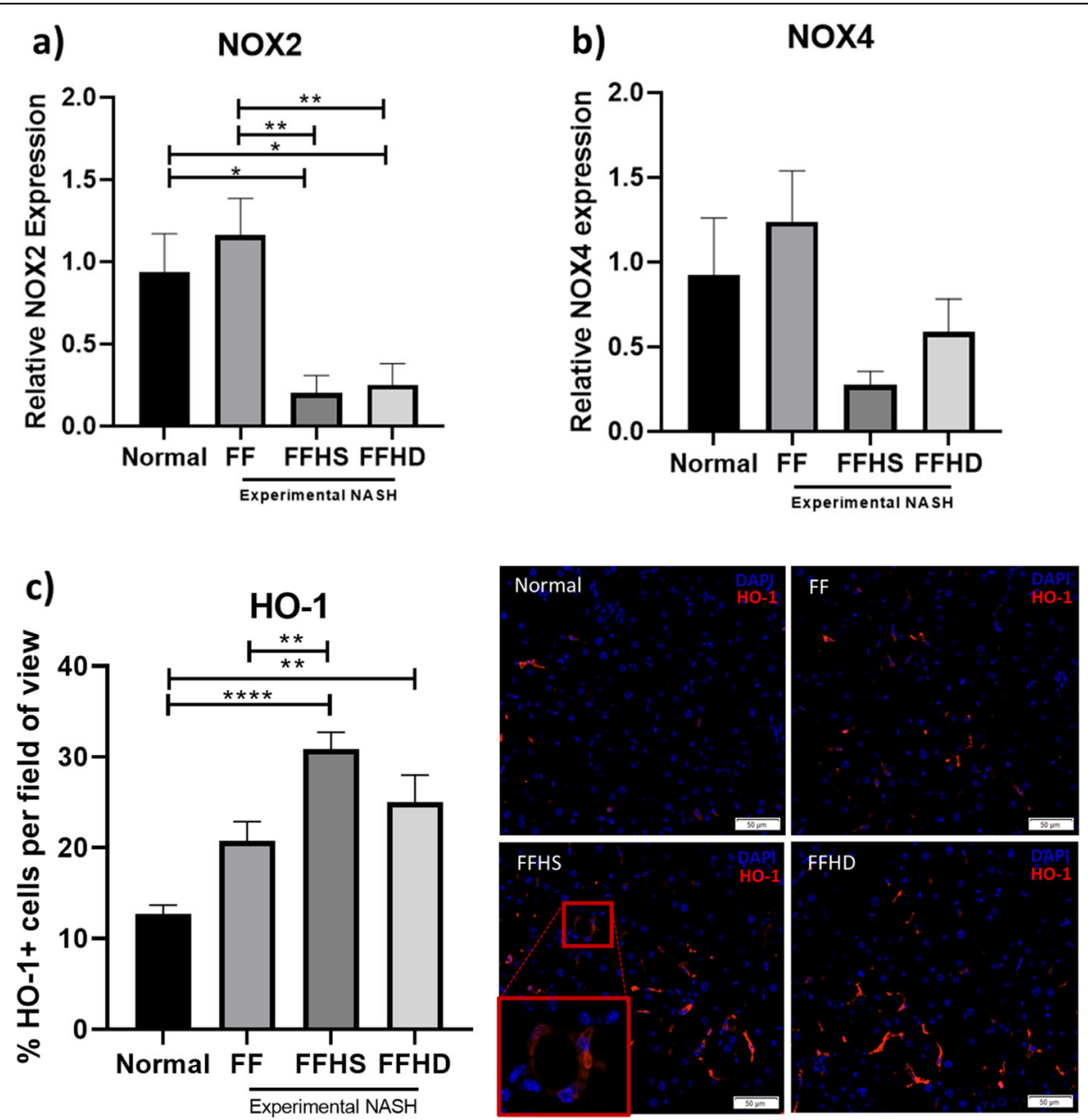

Fig. 4 Antioxidant effects of hAECs in experimental NASH. Compared with normal mice, FFHS and FFHD mice had significantly lower expression levels of Nox2 (a). Within the NASH diet groups, FFHS mice had significantly lower expression levels of Nox2 (a). Nox4 gene expression demonstrated a trend towards lower levels in hAEC exposed mice but did not reach statistical significance $(P=0.10)(\mathbf{b})$. Compared with normal mice, FFHS and FFHD mice had significantly greater levels of HO-1 activity (HO-1+ cells) (c). FFHS mice had significantly higher levels of HO-1 activity compared to untreated FF mice. Magnification: $\times 10$, scale bar $=50 \mu \mathrm{m}$; red box indicating selected area at $\times 20$ magnification. ${ }^{*} P<0.05$, ${ }^{* *} P<0.01$, ${ }^{* * *} P<0.0001$. HO-1, Heme-oxygenase 


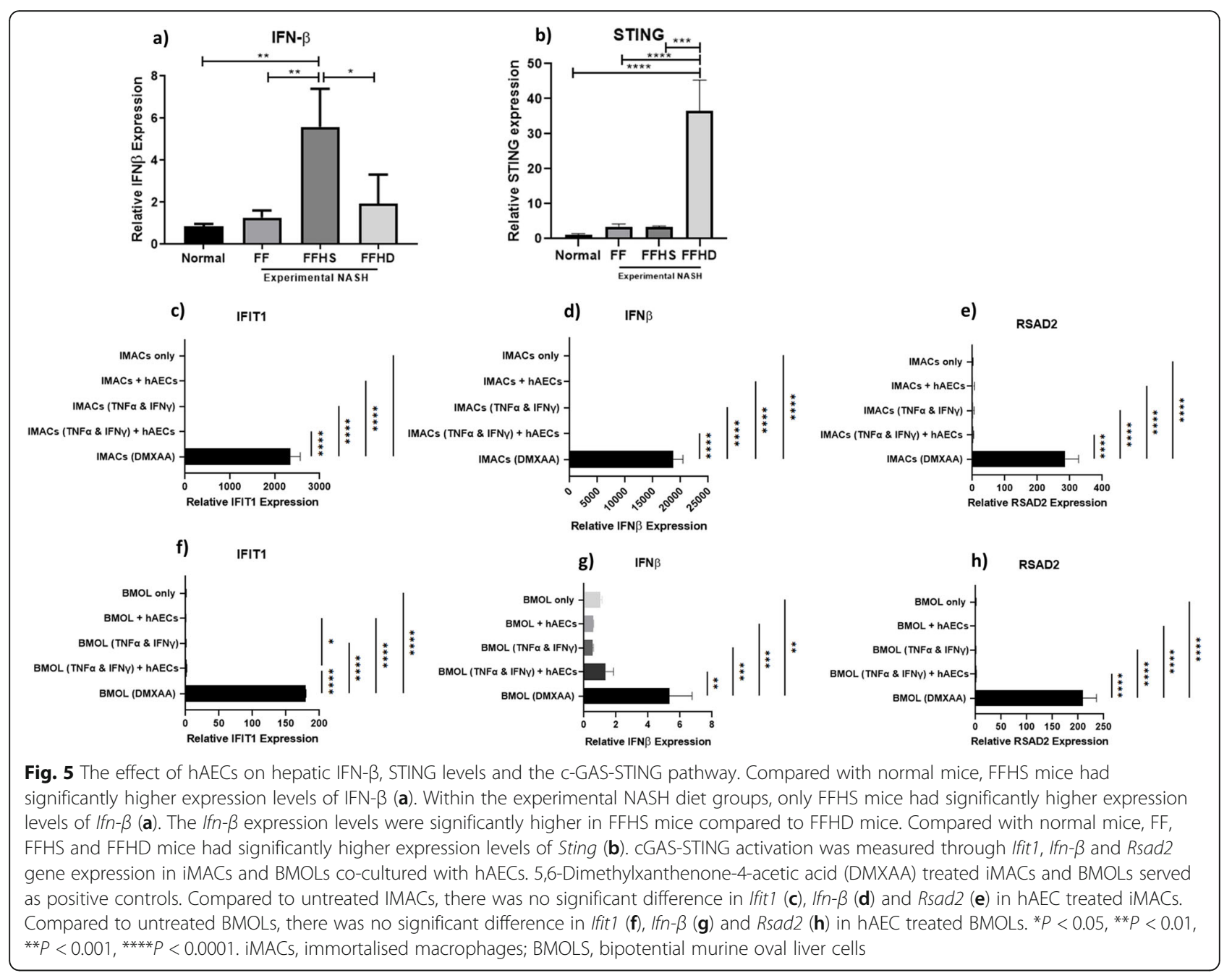

while we observed a potent anti-inflammatory effect of hAECs through the reduction in the LPC mitogens, we saw a lesser effect on the LPC numbers. This indicates that other factors including Wnt ligands recently reported to be produced by hAECs [38] may still persist to drive the LPC response in liver homeostasis and repair [39]. Furthermore, in combination with our recent report that hAECs can promote LPC proliferation and differentiation in vitro and in the absence of inflammatory cytokines [26], these findings support the notion that hAECs support liver repair and reduce inflammation. It is also noteworthy that hAECs reduce LPC mitogens in this context given that uncontrolled proliferation and growth of LPCs can lead to the development of liver cancer [40-42].

We also assessed the impact of hAEC treatment on neutrophil infiltration and activation given the relevance of neutrophils and oxidative stress in both adults with NASH and obese children at risk of developing NASH $[43,44]$. Indeed, pharmaceutical approaches have been employed to address this contributor to NASH progression [31]. The degranulation of neutrophils releases myeloperoxidase (MPO), a ROS-producing enzyme that oxidises phosphatidylcholine, creating a positive feedback loop by activating more neutrophils while also acting as a ligand for scavenger receptors, thus exacerbating fibrogenesis [45]. Here, we observed that the administration of either a single or double dose of hAECs significantly reduced neutrophil infiltration as determined by NIMP-R14 (Ly-6G/-6C) staining which coincided with a reduction in hepatic MPO levels. Since these findings were limited to immunofluorescent analysis it would be beneficial to assess the changes in leukocyte numbers and neutrophil infiltration through flow cytometry in future studies. Further to this, we observed a significant reduction in the expression levels of NADPH oxidase 2 (NOX2) in mice administered with hAECs at both doses, but no significant difference in NOX4 expression levels. NOXs are a family of enzymes known to produce ROS during liver injury [46] and have been implicated in the activation of quiescent HSCs to myofibroblasts [47]. The NOX isoforms are differentially 
expressed by resident liver cells. Hepatic macrophages only express NOX2, while hepatocytes and hepatic stellate cells express both NOX2 and NOX4 as well as other isoforms [47]. The reduction in NOX2 expression levels following hAEC administration may be attributed to reduced macrophage recruitment previously reported [19, $24,48]$. In addition to the reduction in ROS-producing enzymes, hAEC administration also resulted in an increase in the anti-oxidant HO-1, thus suggesting that the reduction in fibrosis, reported in our previous work [24], may have been achieved through a combination of reduced oxidative stress and improved anti-oxidant capacity. Considering the role of ROS in mediating HSC activation, hepatocyte apoptosis, inhibition of hepatocyte replication and accumulation of LPCs [14, 47], these findings are extremely encouraging for the clinical translation of hAEC as a therapy for NASH. The ability of hAECs to reduce oxidative stress that enhances oncogenic mutational events in liver cells; in particular proliferating LPCs, is important as it has been shown that attenuation of the inflammatory response reduces the incidence of HCC in mouse models of fatty liver disease $[49,50]$.

The severity of hepatic inflammation has been identified as an independent risk factor for fibrosis progression in NASH [51]. Interferon $\beta$ (IFN $\beta$ ) was suggested as a potential anti-fibrotic for NASH with its ability to downregulate fibrogenic genes associated with TGF $\beta-1$ and $M y D 88$ pathways [15]. As such, we measured total gene expression of IFN $\beta$ in the livers of NASH mice and observed that a single dose of hAECs significantly increased IFN $\beta$ where a double dose of hAECs failed to achieve this. Given the implication of the c-GAS-STING pathway in the initiation of IFN $\beta$ expression and progression of liver fibrosis $[52,53]$, we then assessed the transcription of STING and IFN-inducible genes in total liver lysates. Here, we observed that STING expression was only significantly increased in the livers of mice exposed to a double dose of hAECs. Furthermore, we did not observe significant expression of common IFN-induced genes in vivo. Next, we performed in vitro studies using immortalised macrophages (iMACs) and a mouse LPC cell line (BMOLs) to further investigate the role of STING signalling. The purpose of this coculture experiment was to investigate the whether the origin of the increased IFN $\beta$ and STING levels was specific to either of these cell populations. The coculture experiment allowed us to investigate the direct effect of the hAECs on the c-GAS-STING pathway on macrophages and liver progenitor cells independent of each other. In this experiment, DMXAA acted as a positive control to activate the cGAS-STING pathway to confirm that the cGAS-
STING pathway could be activated in iMAC and BMOL cell lines. No changes in the expression of Ifn $\beta$, Ifit1 and Rsad 2 genes in iMACs or BMOLs following co-culture with hAECs were observed. hAECs did not lead to IFN induction in vitro and did not result in the initiation of c-GAS-STING signalling. Together, these data do not support a role for hAECs in cGAS-STING activation. Additionally, increased hepatic STING transcription while coincident with the double dose of hAECs does not appear to be a direct consequence of hAEC administration. It is important to recognise that the co-culture studies were limited by use of immortalised macrophages and BMOLs. Future co-culture studies using primary cells or transcriptional profiling of flow sorted cell populations may provide further insights into the cell-cell interactions. cGAS or STING knockout cell lines and/or mouse strains would be beneficial to ascertain the impact of hAECs on the cGAS-STING pathway. This is particularly important given the growing evidence that the DNA-sensing cGAS-STING pathway is critical to NASH progression [52].

As noted previously, we did not show that a second hAEC dose consistently increased efficacy. In this study, our experimental model involved the administration of two doses of hAECs through intraperitoneal injection at week 34 and 38 of the 42 week disease model of NASH. The dosage of $2 \times 10^{6}$ hAECs was chosen based on our previous work in the $\mathrm{CCL}_{4}$ chronic liver injury model [26]. The timepoints for hAEC administration were based on disease progression. By week 34, fibrosis is established in this experimental NASH model and the 4week interval between the first and second dose is based on a published model of $\mathrm{CCL}_{4}$ liver injury [18]. One of the reasons that the repeated hAEC dose did not significantly increase efficacy of the treatment may be due to the long-lasting effects of hAECs, where a single dose of cells may have effects that last for up to 10 weeks post administration [54]. It may not be possible to demonstrate an additive effect if an experimental outcome is maximally suppressed following a single hAEC dose (Fig. 2b-d). In addition, hAEC efficacy may be related to route of administration. Administration of hAECs through intraperitoneal injection was based on previous studies reporting reduced inflammation, fibrosis and activation of neutrophils through this administration route. Cargonini et al. showed that hAECs reduced inflammation and fibrosis of the lungs when they were administered intratracheally, intravenously or intraperitoneally [55]. Further, we had previously reported that intraperitoneally administered hAECs modify infiltration and activation of neutrophils and neutrophil-derived myeloperoxidase via pro-resolution lipid-based mediator, Lipoxin A4 [56]. Future studies focussing on the impact 
of the route of administration on the hAECs mechanism of action in resolving NASH would be beneficial.

\section{Conclusion}

In summary, we provide the first evidence that hAECs reduce the LPC response in experimental NASH. This was likely achieved through a reduction in LPC mitogens as shown in this study and possibly mediated by the reduction in hepatic macrophages observed in previous work [24]. We also report for the first time that hAEC administration reduced inflammation and hepatic levels of ROS-producing enzymes concomitant with increased anti-oxidant capacity. We observed an increase in antifibrotic IFN $\beta$ following hAEC administration that appeared to be independent of cGAS-STING pathway activation. These findings are supportive of clinical translation of hAECs as a therapy for NASH. The differences seen in this study between single versus double doses of hAECs suggests that dose escalation trials should be designed to assess optimal dose rather than maximal tolerable dose. Future studies should also assess the efficacy of repeated doses compared to increased doses.

\section{Abbreviations}

BMOL: Bipotential murine oval liver; DMXAA: 5,6-Dimethylxanthenone-4acetic acid; FF: Fast food diet; hAEC: Human amniotic epithelial cells; HBSS: Hanks Balanced Salt Solution; HCC: Hepatocellular carcinoma; HO1: Heme oxygenase 1; HSC: Hepatic stellate cells; IF: Immunofluorescence; IFN $\beta$ : Interferon $\beta$; IFNY: Interferon $\gamma ;$ IHC: Immunohistochemistry; IL6: Interleukin 6; iMAC: Immortalised mouse macrophages; IP: Intraperitoneal LPC: Liver progenitor cell; LTB: Lymphotoxin $\beta$; MPO: Myeloperoxidase; NAC: N-Acetylcysteine; NAFLD: Non-alcoholic fatty liver disease; NASH: Nonalcoholic steatohepatitis; NOX: NADPH oxidase; ROS: Reactive oxygen species; RT-PCR: Real time quantitative polymerase chain reaction; SAMe: Sadenosylmethionine; SEM: Standard error of mean; TNF: Tumour necrosis factor; TWEAK: Tumour necrosis factor-like weak inducer of apoptosis

\section{Supplementary Information}

The online version contains supplementary material available at https://doi. org/10.1186/s13287-021-02476-6.

Additional file 1: Supplemental Figure 1. Representation of cell purity for clinical isolation of hAECs Isolated hAECs have a cell surface profile of $>90 \%$ EpCAM (a), < 1\% CD90 (b), <1\% CD45 (c), <1\% CD31 (d) positive cells. The red and blue histograms represent the hAEC population and the negative control, respectively (a). The red and blue histograms represent the hAEC population and the positive control, respectively $(b-d)$.

\section{Acknowledgements \\ The authors thank Associate Professor Ashley Mansell (Hudson Institute of Medical Research, Australia) for his kind gift of iMAC cells. The authors acknowledge the facilities and scientific and technical assistance of the Monash Health Translation Precinct (MHTP) Medical Genomics Facility, the MHTP Monash Micro Imaging Platform, the MHTP Monash Animal Research Platform (MARP) and the Monash Histology Platform, Department of Anatomy and Developmental Biology, Monash University.}

\section{Authors' contributions}

$M G, N K$, JC and $A H$ performed the experiments. MG and RL analysed the data. RL and MPG conceived and designed all the experiments. MG and RL wrote the manuscript. RL, GM and WS were responsible for the grant application. RL, GM, GY and WS edited the manuscript. All authors have read and approved the final manuscript.

\section{Funding}

This work was supported by an MRFF Stem Cell Mission grant (APP1201805).

Availability of data and materials

The datasets used and analysed during the current study are available from the corresponding author on request.

\section{Declarations}

Ethics approval and consent to participate

This animal study was approved by Monash University Animal Ethics Committee (AE\# B13/01) and conducted in accordance with the Australian Code of Practice for the Care and Use of Animals for Scientific Purposes (2006). The Monash Health Human Research Ethics Committee approved the collection and use of human amnion (Monash Health HREC approval numbers: 01067B, 12223B). Informed written consent was obtained from each patient prior to surgery.

\section{Consent for publication}

Not applicable.

\section{Competing interests}

The authors declare that they have no competing interests.

\section{Author details}

${ }^{1}$ Centre for Inflammatory Disease, School of Clinical Sciences, Monash University, Melbourne, Victoria, Australia. ${ }^{2}$ The Ritchie Centre, Hudson Institute of Medical Research, Melbourne, Victoria, Australia.

${ }^{3}$ Gastroenterology and Hepatology Unit, Monash Health, Melbourne, Victoria, Australia. ${ }^{4}$ Department of Molecular and Translational Science, Monash University, Clayton, Victoria, Australia. ${ }^{5}$ Centre for Innate Immunity and Infectious Disease, Hudson Institute of Medical Research, Melbourne, Victoria, Australia. ${ }^{6}$ Centre for Medical Research, Harry Perkins Institute of Medical Research, QEIl Medical Centre, Nedlands, Western Australia, Australia. ${ }^{7}$ School of Molecular Sciences, The University of Western Australia, Crawley, Western Australia, Australia. ${ }^{8} \mathrm{Centre}$ for Cell Therapy and Regenerative Medicine, School of Biomedical Sciences, The University of Western Australia, Crawley, Western Australia, Australia. ${ }^{9}$ Department of Obstetrics and Gynaecology, Monash University, Melbourne, Victoria, Australia.

Received: 26 October 2020 Accepted: 26 June 2021

Published online: 28 July 2021

\section{References}

1. Cotter TG, Rinella M. Nonalcoholic fatty liver disease 2020: the state of the disease. Gastroenterology (New York, N.Y. 1943). 2020;158(7):1851-64.

2. Torres DM, Williams CD, Harrison SA. Features, diagnosis, and treatment of nonalcoholic fatty liver disease. Clin Gastroenterol Hepatol. 2012;10(8):83758. https://doi.org/10.1016/j.cgh.2012.03.011.

3. Singh $\mathrm{S}$, et al. Fibrosis progression in nonalcoholic fatty liver vs nonalcoholic steatohepatitis: a systematic review and meta-analysis of paired-biopsy studies. Clin Gastroenterol Hepatol. 2015;13(4):643-654.e9.

4. Younossi ZM, Koenig AB, Abdelatif D, Fazel Y, Henry L, Wymer M. Global epidemiology of nonalcoholic fatty liver disease-meta-analytic assessment of prevalence, incidence, and outcomes. Hepatology. 2016;64(1):73-84. https://doi.org/10.1002/hep.28431.

5. Knight B, Lim R, Yeoh GC, Olynyk JK. Interferon-y exacerbates liver damage, the hepatic progenitor cell response and fibrosis in a mouse model of chronic liver injury. J Hepatol. 2007;47(6):826-33. https://doi.org/10.1016/j. jhep.2007.06.022.

6. Lowes KN, Brennan BA, Yeoh GC, Olynyk JK. Oval cell numbers in human chronic liver diseases are directly related to disease severity. Am J Pathol. 1999;154(2):537-41. https://doi.org/10.1016/50002-9440(10)65299-6.

7. Nguyen LN, Furuya MH, Wolfraim LA, Nguyen AP, Holdren MS, Campbell JS, et al. Transforming growth factor-beta differentially regulates oval cell and hepatocyte proliferation. Hepatology. 2007:45(1):31-41. https://doi.org/10.1 002/hep.21466. 
8. Yeoh GC, et al. Opposing roles of gp130-mediated STAT-3 and ERK-1/ 2 signaling in liver progenitor cell migration and proliferation. Hepatology. 2007;45(2):486-94. https://doi.org/10.1002/hep.21535.

9. Best J, Manka P, Syn WK, Dollé L, van Grunsven L, Canbay A. Role of liver progenitors in liver regeneration. Hepatobiliary Surg Nutr. 2015;4(1):48-58. https://doi.org/10.3978/j.issn.2304-3881.2015.01.16.

10. Marquardt JU. Deconvolution of the cellular origin in hepatocellular carcinoma: hepatocytes take the center stage. Hepatology. 2016;64(4):10203. https://doi.org/10.1002/hep.28671.

11. Emel Pamuk G, Sonsuz A. N-Acetylcysteine in the treatment of nonalcoholic steatohepatitis. J Gastroenterol Hepatol. 2003;18(10):1220-1. https://doi.org/10.1046/j.1440-1746.2003.03156.x.

12. Gulbahar $\mathrm{O}$, et al. Treatment of non-alcoholic steatohepatitis with $\mathrm{N}$-acetyl cystein. Gastroenterology (New York, N.Y. 1943). 2000;118(4):A1444.

13. Oz HS, Im HJ, Chen TS, de Villiers WJS, McClain CJ. Glutathione-enhancing agents protect against steatohepatitis in a dietary model. J Biochem Mol Toxicol. 2006:20(1):39-47. https://doi.org/10.1002/jbt.20109.

14. Roskams T, Yang SQ, Koteish A, Durnez A, DeVos R, Huang X, et al. Oxidative stress and oval cell accumulation in mice and humans with alcoholic and nonalcoholic fatty liver disease. Am J Pathol. 2003;163(4): 1301-11. https://doi.org/10.1016/S0002-9440(10)63489-X.

15. Shimozono R, Nishimura K, Akiyama H, Funamoto S, Izawa A, Sai T, et al. Interferon- $\beta$ mediates signaling pathways uniquely regulated in hepatic stellate cells and attenuates the progression of hepatic fibrosis in a dietary mouse model. J Interferon Cytokine Res. 2015;35(6):464-73. https://doi.org/1 0.1089/jir.2014.0096.

16. Lim R, Hodge A, Moore G, Wallace EM, Sievert W. A pilot study evaluating the safety of intravenously administered human amnion epithelial cells for the treatment of hepatic fibrosis. Front Pharmacol. 2017;8:549. https://doi. org/10.3389/fphar.2017.00549.

17. Lim R, Malhotra A, Tan J, Chan ST, Lau S, Zhu D, et al. First-in-human administration of allogeneic amnion cells in premature infants with bronchopulmonary dysplasia: a safety study. Stem Cells Transl Med. 2018; 7(9):628-35. https://doi.org/10.1002/sctm.18-0079.

18. Manuelpillai U, Lourensz D, Vaghjiani V, Tchongue J, Lacey D, Tee JY, et al. Human amniotic epithelial cell transplantation induces markers of alternative macrophage activation and reduces established hepatic fibrosis. PLoS One. 2012;7(6):e38631. https://doi.org/10.1371/journal.pone. 0038631.

19. Tan JL, Chan ST, Wallace EM, Lim R. Human amnion epithelial cells mediate lung repair by directly modulating macrophage recruitment and polarization. Cell Transplant. 2014;23(3):319-28. https://doi.org/10.3727/0963 $68912 \times 661409$.

20. Song Y-S, Joo HW, Park IH, Shen GY, Lee Y, Shin JH, et al. Transplanted human amniotic epithelial cells secrete paracrine proangiogenic cytokines in rat model of myocardial infarctio. Cell Transplant. 2015;24(10):2055-64. https://doi.org/10.3727/096368914X685609.

21. Zhao B, Liu JQ, Yang C, Zheng Z, Zhou Q, Guan H, et al. Human amniotic epithelial cells attenuate TGF- $\beta 1$-induced human dermal fibroblast transformation to myofibroblasts via TGF- $\beta 1 /$ Smad3 pathway. Cytotherapy. 2016:18(8):1012-24. https://doi.org/10.1016/j.jcyt.2016.04.009.

22. Leaw B, Zhu D, Tan J, Muljadi R, Saad MI, Mockler JC, et al. Human amnion epithelial cells rescue cell death via immunomodulation of microglia in a mouse model of perinata brain injury. Stem Cell Res Ther. 2017;8(1):46. https://doi.org/10.11 86/s13287-017-0496-3

23. Alhomrani M, Correia J, Zavou M, Leaw B, Kuk N, Xu R, et al. The human amnion epithelial cell secretome decreases hepatic fibrosis in mice with chronic liver fibrosis. Front Pharmacol. 2017;8. https://doi.org/10.3389/fphar.2 017.00748 .

24. Kuk N, Hodge A, Sun Y, Correia J, Alhomrani M, Samuel C, et al. Human amnion epithelial cells and their soluble factors reduce liver fibrosis in murine non-alcoholic steatohepatitis. J Gastroenterol Hepatol. 2019;34(8): 1441-9. https://doi.org/10.1111/jgh.14643.

25. Manuelpillai U, Tchongue J, Lourensz D, Vaghjiani V, Samuel CS, Liu A, et al. Transplantation of human amnion epithelial cells reduces hepatic fibrosis in immunocompetent $\mathrm{CCl}_{4}$-treated mice. Cell Transplant. 2010;19(9):1157-68. https://doi.org/10.3727/096368910X504496.

26. Hodge A, et al. Human amnion epithelial cells produce soluble factors that enhance liver repair by reducing fibrosis while maintaining regeneration in a model of chronic liver injury. Cell Transplant. 2020;29:963689720950221.

27. Sant'Anna LB, et al. Amniotic membrane application reduces liver fibrosis in a bile duct ligation rat model. Cell Transplant. 2011;20(3):441-53. https://doi. org/10.3727/096368910X522252.

28. Andrewartha N, Yeoh G. Human amnion epithelial cell therapy for chronic liver disease. Stem Cells Int. 2019;2019:8106482.

29. Miki T, et al. Isolation of amniotic epithelial stem cells. Curr Protoc Stem Cell Biol. 2010;Chapter 1:Unit 1E.3.

30. Livak KJ, Schmittgen TD. Analysis of relative gene expression data using real-time quantitative PCR and the 2(-Delta Delta C(T)) Method. Methods. 2001;25(4):402-8. https://doi.org/10.1006/meth.2001.1262.

31. Bartneck $M$, Wang J. Therapeutic targeting of neutrophil granulocytes in inflammatory liver disease. Front Immunol. 2019;10:2257. https://doi.org/1 0.3389/fimmu.2019.02257.

32. Gressner AM, Lahme B, Mannherz HG, Polzar B. TGF-beta-mediated hepatocellular apoptosis by rat and human hepatoma cells and primary rat hepatocytes. J Hepatol. 1997;26(5):1079-92. https://doi.org/10.1016/S0168-82 78(97)80117-1.

33. Oberhammer F, Bursch W, Parzefall W, Breit P, Erber E, Stadler M, et al. Effect of transforming growth factor beta on cell death of cultured rat hepatocytes. Cancer Res. 1991;51(9):2478-85.

34. Dwyer BJ, et al. TWEAK and LTB Signaling during chronic liver disease. Front Immunol. 2014;5:39.

35. Akhurst B, et al. Differential lymphotoxin-beta and interferon gamma signaling during mouse liver regeneration induced by chronic and acute injury. Hepatology (Baltimore, Md.). 2005;41(2):327.

36. Knight B, Yeoh G. TNF/LTa double knockout mice display abnormal inflammatory and regenerative responses to acute and chronic liver injury. Cell Tissue Res. 2005;319(1):61-70. https://doi.org/10.1007/s00441004-1003-6.

37. Knight B, Yeoh GCT, Husk KL, Ly T, Abraham LJ, Yu C, et al. Impaired preneoplastic changes and liver tumor formation in tumor necrosis factor receptor type 1 knockout mice. J Exp Med. 2000;192(12):1809-18. https:// doi.org/10.1084/jem.192.12.1809.

38. Zhu D, Kusuma GD, Schwab R, Chan ST, Tan J, Saad MI, et al. Prematurity negatively affects regenerative properties of human amniotic epithelial cells in the context of lung repair. Clin Sci. 2020;134(20):2665-79. https://doi. org/10.1042/CS20200859.

39. Russell JO, Monga SP. Wnt/ $\beta$-catenin signaling in liver development, homeostasis, and pathobiology. Annu Rev Pathol. 2018;13(1):351-78. https://doi.org/10.1146/annurev-pathol-020117-044010.

40. Lee K-P, Lee JH, Kim TS, Kim TH, Park HD, Byun JS, et al. The Hippo-Salvador pathway restrains hepatic oval cell proliferation, liver size, and liver tumorigenesis. Proc Natl Acad Sci U S A. 2010;107(18):8248-53. https://doi. org/10.1073/pnas.0912203107.

41. Libbrecht L, Desmet V, Damme BV, Roskams T. The immunohistochemical phenotype of dysplastic foci in human liver: correlation with putative progenitor cells. J Hepatol. 2000;33(1):76-84. https://doi.org/10.1016/S01688278(00)80162-2.

42. Tummala KS, Brandt M, Teijeiro A, Graña O, Schwabe RF, Perna C, et al. Hepatocellular carcinomas originate predominantly from hepatocytes and benign lesions from hepatic progenitor cells. Cell Rep. 2017;19(3):584-600. https://doi.org/10.1016/j.celrep.2017.03.059.

43. Strauss RS. Comparison of serum concentrations of alphatocopherol and beta-carotene in a cross-sectional sample of obese and nonobese children (NHANES III). National Health and Nutrition Examination Survey. J Pediatr. 1999;134(2):160-5. https://doi.org/10.1 016/S0022-3476(99)70409-9.

44. Inzaugarat ME, Ferreyra Solari NE, Billordo LA, Abecasis R, Gadano $A C$, Cherñavsky AC. Altered phenotype and functionality of circulating immune cells characterize adult patients with nonalcoholic steatohepatitis. J Clin Immunol. 2011;31(6):1120-30. https://doi.org/10.1007/s10875-011-9571-1.

45. Ikura Y, Ohsawa M, Suekane T, Fukushima H, Itabe H, Jomura H, et al. Localization of oxidized phosphatidylcholine in nonalcoholic fatty liver disease: impact on disease progression. Hepatology. 2006;43(3):506-14. https://doi.org/10.1002/hep.21070.

46. Paik Y-H, Kim J, Aoyama T, de Minicis S, Bataller R, Brenner DA. Role of NADPH oxidases in liver fibrosis. Antioxid Redox Signal. 2014;20(17):2854-72. https://doi.org/10.1089/ars.2013.5619. 
47. Liang S, Kisseleva T, Brenner DA. The role of NADPH oxidases (NOXs) in liver fibrosis and the activation of myofibroblasts. Front Physiol. 2016;7:17.

48. Hodge $A$, et al. oluble factors derived from human amniotic epithelial cells suppress collagen production in human hepatic stellate cells. Cytotherapy (Oxford, England). 2014;16(8):1132-44.

49. Passman AM, Strauss RP, McSpadden S, Finch-Edmondson ML, Woo KH, Diepeveen LA, et al. A modified choline-deficient, ethioninesupplemented diet reduces morbidity and retains a liver progenitor cell response in mice. Dis Model Mech. 2015;8(12):1635-41. https:// doi.org/10.1242/dmm.022020.

50. Viebahn CS, Benseler V, Holz LE, Elsegood CL, Vo M, Bertolino P, et al. Invading macrophages play a major role in the liver progenitor cell response to chronic liver injury. J Hepatol. 2010;53(3):500-7. https://doi. org/10.1016/j.jhep.2010.04.010.

51. Argo CK, Northup PG, al-Osaimi AMS, Caldwell SH. Systematic review of risk factors for fibrosis progression in non-alcoholic steatohepatitis. J Hepatol. 2009;51(2):371-9. https://doi.org/10.1016/j. jhep.2009.03.019.

52. Luo $X$, et al. Expression of STING is increased in liver tissues from patients with NAFLD and promotes macrophage-mediated hepatic inflammation and fibrosis in mice. Gastroenterology (New York, N.Y. 1943). 2018;155(6): 1971-1984.e4

53. Yu Y, Liu Y, An W, Song J, Zhang Y, Zhao X. STING-mediated inflammation in Kupffer cells contributes to progression of nonalcoholic steatohepatitis. J Clin Invest. 2019;129(2):546-55. https://doi.org/10.1172/JC1121842.

54. Zhu D, Tan J, Maleken AS, Muljadi R, Chan ST, Lau SN, et al. Human amnion cells reverse acute and chronic pulmonary damage in experimental neonatal lung injury. Stem Cell Res Ther. 2017;8(1):257. https://doi.org/10.11 86/s13287-017-0689-9.

55. Cargnoni A, Gibelli L, Tosini A, Signoroni PB, Nassuato C, Arienti D, et al. Transplantation of allogeneic and xenogeneic placenta-derived cells reduces bleomycin-induced lung fibrosis. Cell Transplant. 2009;18(4):405-22. https://doi.org/10.3727/096368909788809857.

56. Tan JL, Tan YZ, Muljadi R, Chan ST, Lau SN, Mockler JC, et al. Amnion epithelial cells promote lung repair via Lipoxin A(4). Stem Cells Transl Med. 2017;6(4):1085-95. https://doi.org/10.5966/sctm.2016-0077.

\section{Publisher's Note}

Springer Nature remains neutral with regard to jurisdictional claims in published maps and institutional affiliations.

Ready to submit your research? Choose BMC and benefit from:

- fast, convenient online submission

- thorough peer review by experienced researchers in your field

- rapid publication on acceptance

- support for research data, including large and complex data types

- gold Open Access which fosters wider collaboration and increased citations

- maximum visibility for your research: over $100 \mathrm{M}$ website views per year

At $\mathrm{BMC}$, research is always in progress.

Learn more biomedcentral.com/submissions 